\title{
The conceptual, cunning and conclusive experiment in psychology
}

major revision (16 April 2021)

\author{
Stijn Debrouwere \\ Yves Rosseel \\ Ghent University (Belgium) \\ stijn.debrouwere@ugent.be
}

\begin{abstract}
The ideal experiment in physics must be conceptual, cunning and conclusive. Adoption of these same standards in psychology has led to experiments that are uninformative and frivolous. We explain why we believe that psychology is fundamentally incompatible with hypothesis-driven theoretical science and conclude that this erodes the logic behind recent proposals to improve psychological research, such as stricter statistical standards, preregistration and replication. The failure of psychology is not that it is somehow insufficiently scientific, but rather that it is inordinately fond of methods that are a mismatch for the aspirations of researchers in the field, at the expense of valuable empirical research.
\end{abstract}

\section{Contents}

Introduction . . . . . . . . . . . . . . . . . . . . . 1

Why bold predictions do not lead to conclusive evidence in psychology . . . . . 3

Psychology as an inductive science . . . . . . . . . . . . . . 6

Final thoughts . . . . . . . . . . . . . . . . . . . 12

Acknowledgements . . . . . . . . . . . . . . . . . 13

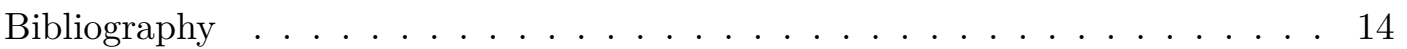

\section{Introduction}

A psychologist wonders whether people eat more when portions look smaller, and with some tinkering is able to engineer a soup bowl that surreptitiously replenishes itself. It does in fact cause people to eat more soup. An ingenious manipulation.

A psychologist wonders whether the mental pathways we use to assess risks in everyday situations might break down when we try to reason about abstract probabilities. Perhaps they will, as natural selection is frugal and does not favor general-purpose neural machinery. 
An online survey finds that test subjects have considerable probabilistic aptitude, but falter whenever a problem is devoid of context. Another win for evolutionary psychology.

A psychologist wonders what comes first, thoughts or feelings, so she has people take either a leisurely stroll or a brisk run before meeting with a handsome experimenter, then compares the level of arousal in both groups. The average level of arousal is higher among those who ran and the difference between groups is larger than two standard errors. Proof that feelings are post-hoc interpretations of a pre-existing bodily state.

Seen from one perspective, these three vignettes are evidence of a mature psychological science: one that knows how to unravel the mysteries of human behavior through cunning interventions, one that is not content to catalogue human behavior but instead seeks theories that might explain it, one that uses statistical inference to ensure experiments are not merely suggestive, but lend firm support to the hypothesis at hand.

Seen from another perspective, these vignettes represent an experimental science whose interventions lack ecological validity, a science that theorizes freely but misses some of the basic facts, a science that uses statistics to whitewash uncertainty.

Psychology has a rich history of randomized experiments, produces ever more refined instruments and evaluates research using complex statistical models. In that sense, it is psychology and not economics that deserves to be called queen of the social sciences. Yet, rather suspiciously, hypotheses are always corroborated, no model ever cast aside (Lykken 1991). Even after a century of experiments and statistical tests, we still disagree about which theories should count as the field's crowning achievements, or whether any such achievements exist (Wade 1982; Mischel 2008). The literature mentions many theories, but each theory is mentioned rarely (McPhetres et al. 2021). The most-cited figures in introductionary textbooks are historical ones like Sigmund Freud with no relevance to current research (Griggs and Christopher 2016). Psychology eschews the chaos of field research in favor of careful and repeatable interventions in the lab. Except that our experiments turn out differently when we try again (Open Science Collaboration 2015) and those few experiments that have achieved pop culture status, like Zimbardo's prison experiment and Rosenhan's infiltration into psychiatric wards, are fraudulent (Blum 2018; Cahalan 2019).

Cunning interventions, theoretical sophistication and mathematical leverage fuel the successes of physics with almost "unreasonable effectiveness" (Wigner 1960), and one might think such methods ought to do the same for psychology. They do not because, in psychology, theories are not clearly tied to observational outcomes, hypotheses cannot discriminate between theories and conclusions from the lab do not translate to scenarios of intrinsic interest. Under such conditions, hypothesis-driven theoretical science is impossible.

The failure of psychology is not that it is somehow insufficiently scientific, but rather that it is inordinately fond of methods that are a mismatch for the aspirations of researchers in the field. We will show how hypothetico-deductive reasoning in psychology falls short, and then explore what a more empirical psychology might look like. 


\section{Why bold predictions do not lead to conclusive evidence in psychology}

Research in experimental psychology proceeds by and large as it does in any natural science: make an educated guess at the gears and pulleys that drive a phenomenon of interest, extract from this blueprint a tangible if-this-then-that hypothesis, set up an experiment that might corroborate or falsify the hypothesis by means of a tangible prediction, collect and analyze the data, conclude against or in favor of our tentative explanation.

At the core of this method is a quid pro quo: make predictions that are extremely unlikely to hold in general yet flow naturally from your candidate theory, and in exchange you will not have to inductively compile an endless book of proof, but instead may take a small handful of positive instances to deductively establish the truth of a causal or structural explanation.

The inductive scientist might note: "Never in all the years that I have lived have I seen ice sink in water, so I guess that it never does. Here's a million row spreadsheet for proof." The deductive scientist may instead report: "Lighter substances float, denser ones sink. Ice is lighter than water, hence ice will float on water. Here's a quick demonstration." Induction produces generalizations, whereas deduction banks on theories.

Harvey Fletcher and Robert Millikan's careful measurement of tiny ionized oil drops conclusively showed that electric charge is always precisely a multiple of some basic unit, thought to be the charge of a single electron (Perry 2007). There is no reason to distrust the setup and no other plausible explanation, so this experiment established once and for all the existence of the "minimum quantity of electricity" that Johnstone Stoney hypothesized in the late 19th century as an explanation for the unique emission spectrum of different gases (Stoney 1894).

When it works, hypothetico-deductive inference is a brilliant epistemic trick that produces large amounts of information from small amounts of observational feedstock. Physicist John Platt labeled it strong inference, "For exploring the unknown, there is no faster method; this is the minimum sequence of steps." (Platt 1964, 347) Philosopher of science Larry Laudan thought it was so wonderful he named it aristocratic induction (Laudan 1981, ch. 6), as opposed to laborious plebeian induction. With aristocratic induction, there is no need to go to Charles Darwinesque lengths to observe nature for years on end before one may venture a guess as to how it all fits together. Instead, a stroke of insight and one well-placed observation could be enough to validate a theory.

Basic science in physics possesses unique epistemic and social features that render the hypothetico-deductive mode of science not merely possible but fruitful:

- theories make precise predictions which are by definition bold predictions, as the probability of any point within an infinite sample space is technically zero,

- experimental setup creates a closed system where we may test predictions and expect these predictions to hold unconditionally,

- social standards discourage corrections, auxiliary variables and other ad hoc adjustments to theories and encourage healthy dispute (Campbell 1979),

- ubiquitous links between theories help keep a tight rein on fanciful theorizing, as ad hoc adjustments in one place are likely to wreak havoc and lead to faulty predictions elsewhere (Meehl 1978) 
In such an environment, hypothesis testing works exactly as historians and philosophers such as William Whewell and Karl Popper said it ought to: subject theories to rigorous tests and quickly dispatch of theories that make faulty predictions, with the minimum amount of fuss.

Psychologists often take it for granted that they too may use the hypothetico-deductive mode. We believe this to be false. In psychology, predictions are loosely connected to hypotheses. Hypotheses are loosely connected to theories. Post-hoc modifications to evidence, theory and hypothesis are common. As such, it is impossible for experimental evidence in psychology to truly favor one theory over another.

\section{Predictions only loosely connected to hypotheses.}

When Heinrich Hertz sought to test James Clerk Maxwell's hypothesis that light was a form of electromagnetic radiation, he did so through a series of experiments that proved the existence of electromagnetic waves, that they move at the speed of light and that they "exhibit lightlike behaviors of reflection, refraction, diffraction, and polarization" (Rautio 2014). Hertz did not set out merely to show the existence of electromagnetic waves, but also that these waves had all the qualities Maxwell thought they should have, and that any measurable quantities did not deviate from Maxwell's equations.

In the work of Hertz and in other famous experiments from physics, multiple precise predictions are made. In psychology, predictions tend to be solitary and vague. Solitary, because predictions in psychology postulate one particular effect rather than multiple conditions that each must hold. Vague, because predictions in psychology postulate quality but not quantity: they forecast that a relationship between two variables will be found, but not the magnitude of that relationship.

In theoretical physics, measurement is aimed at verification. In experimental psychology, measurement is aimed edification, at informing us about the magnitude of an effect. This, too, shows two very different approaches to science. The goal of the famous Eddington expeditions to Príncipe and Brazil during a solar eclipse in 1919 was not to investigate, empirically, how light from distant stars is deflected by the gravity of the sun and add this to our repository of physical facts, because Einstein's theory already told us what kind of deflection to expect in this scenario - a little over 0.000483 degrees. Instead, the point was simply to verify whether or not this angle matched observation. (Coles 2019)

To predict specific magnitudes is generally out of reach for psychology, but psychologists do have other methods at their disposal that might strengthen the case for their particular theory. Mediation analysis narrows down the predictive leeway of experiments in psychology by requiring one to specify the mechanism that links two variables together, not just that a relationship exists. Experimental psychologists often raise the stakes by testing a hypothesis through not one but a series of experiments with different stimuli and subjects. Scales can be subjected to psychometric scrutiny before use. Experiments in psychology tend to be fairly small whereas statistical significance becomes more likely with $\sqrt{n}$, so that in practice a minimal effect size is implicitly a part of the hypothesis.

At the same time, qualitative research in physics is common, especially in the context of discovery. And physicists do also rely on a handful of fundamental quantities they must estimate and cannot predict, for example the mass of the Higgs boson and other quantum 
particles.

Nevertheless, predictions in physics and predictions in psychology tend to be on opposite sides of the evidentiary spectrum. It does not help the case of psychology that, in practice, mediation analysis is extremely brittle (Bullock, Green, and Ha 2010), complex models are judged using weak indicators of success such as statistical goodness of fit (Roberts and Pashler 2000) and multiple-study articles are often underpowered and prone to abuse (Schimmack 2012), producing fewer falsifications than simpler statistical methods, not more.

The unbridgeable chasm between physics and psychology appears most vividly in statistical testing. In psychology, we aim to reject a precisely quantified null hypothesis in favor of its unquantified complement, anything not null. In physics, the aim is generally to confirm the null hypothesis (our theory) and reject the alternative hypothesis (any other theory). The latter approach leads to bold conjectures whereas the former provides only very meager evidence in favor of the theory under investigation.

\section{Hypotheses only loosely connected to theories.}

A psychologist may posit that someone with high social standing will tend to do what they can to maintain that standing (our theory) and that they might therefore be inclined to boycott social competitors or withhold access to important resources (our hypothesis). However, if it turns out that instead, those of high social standing tend to be magnanimous, this too is easily explained: generosity is a great way to showcase one's superior status. Our theory aligns equally well with both hypotheses, even though they are diametrically opposed to each other.

When hypotheses are only loosely connected to their parent theory, the hypothetico-deductive method does not apply because our observations are not evidence for a theory and against others. When mutually exclusive hypotheses all fit the theory equally well, or alternatively when mutually exclusive theories all fit the same hypothesis equally well, data from an experiment that lends support to a hypothesis will not transitively lend support to the theory that inspired it.

In psychology, often it's not quite clear what if anything a theory implies for the empirical world. In the worst case, lack of clear implications signals lack of scientific rigor: if a theory does not predict some and rule out other states of the world, it cannot be falsified, and hence it is not scientific. You may recall this was the essence of Karl Popper's objection to early twentieth-century psychoanalysis, which seemed to have an explanation for everything. Our own critique is more modest. Indeterminacy of outcomes and under-identification of causes are not a death sentence to the scientific investigation of mind and behavior, as we will explain later, but they do preclude the hypothetico-deductive method.

\section{Theories only loosely connected to other theories.}

In his reflections on 19th century science, William Whewell concluded that while successful predictions are essential to science, what is really impressive is science's consilience and coherence: how a theory may inform cases beyond its original scope and how different theories dovetail with each other, respectively. Newton's law of gravity applies not just to apples but also to planets, and ties in nicely with his laws of motion. Knowledge does not stand alone, but fits together. 
While it is common for experimental psychologists to claim to contribute to a particular research program or to start from a particular framework such as Daniel Kahneman's heuristics and biases, in practice results from one psychological experiment rarely if ever make us question the validity of other, unrelated experiments, and theories themselves rarely clash with each other. Admittedly not every theory in physics is wedded to every other, and so we may explain the working of some kinds of laser beams while blissfully unaware of high-energy physics or the behavior of gases, but any theory in mechanics that does not conserve energy will clash with everything we know from thermodynamics. In psychology, such restrictions rarely apply. One may hold the most outlandish views on intelligence without gainsaying prevailing views on working memory, childhood learning, language acquisition and much more.

As a result of these weak to non-existent links between theories, experimental psychologists cannot avail themselves of a kind of sanity check that is very important in physics and that in physics is performed well in advance of any sort of experimental verification: should $I$ even bother to test this idea, or would it be nonsensical given everything else we know? In psychology, there are very few hypotheses that one would have to reject prima facie for being incompatible with firmly grounded knowledge about human nature, and in fact journals tend to encourage research that at first blush seems to have a counterintuitive premise, because it fits well with the scientist's rebellious self-image of not succumbing to taboo or conventional wisdom.

Theories in physics are like a house of cards, in precarious balance. Precisely because of that fragility, its body of existing knowledge forms an important safeguard against bad science. Related theories in psychology more closely resemble a spider web, in which any thread may snap without perturbing the whole. Because of psychology's resilience, its body of existing knowledge is overaccommodating to newcomers and overindulgent of their flaws, which harms its scientific credibility.

Because (1) predictions are loosely connected to hypotheses, (2) hypotheses are loosely connected to theories and (3) theories are loosely connected to other theories, psychology gets very little mileage out of theoretical developments and cannot use experiments to separate good theories from bad ones.

Without a convincing connection between theory and hypothesis, hypothesis and prediction, prediction and observation, we are back where we started: the conditions for successful application of the hypothetico-deductive mode are not fulfilled and we must default to what Larry Laudan calls plebeian induction, in which we laboriously enumerate positive instances of our claim under a variety of circumstances until, statistically, it counts as sufficient evidence. Plebeian induction takes more time, but it makes fewer demands: its applicability is limited only by our persistence.

\section{Psychology as an inductive science}

Faced with psychology's poor track record as a hypothetico-deductive science, we may try to find our way out of the swamp by simply doing better. Anne Scheel and colleagues have argued in this journal that while in practice psychologists often "cut corners when testing hypotheses" (Scheel et al. 2020, 5), in principle and with a lot of hard work it is possible for psychological 
research to be every bit as tough-minded as physical research, with validated instruments, proper causal models, well-specified boundary conditions for where the phenomenon will and won't be found, triple-checked auxiliary assumptions and principled statistics. This is a widely shared view among psychologists (Gigerenzer 2010; Muthukrishna and Henrich 2019; Oberauer and Lewandowsky 2019; Borghi and Fini 2019; Fried 2020; Burghardt and Bodansky 2021).

But what happens if a smart, well-considered question nevertheless does not permit strong inference? For example, pediatricians and developmental psychologists believe babies become increasingly fussy sleepers at around 6 months because of the infant's newly acquired gift of object permanence: the child now realizes that when a parent leaves the room, they're actually still around somewhere, and this realization causes distress. It's a plausible explanation. Suppose, however, that we didn't already know the explanandum. Object permanence could then just as well be invoked to hypothesize that babies at the half year mark will become more relaxed and better sleepers: they know that even when they don't see you, you're still around, a soothing thought. It's not clear how we could formalize this into a falsifiable theory amenable to a strong hypothesis test. Does that mean the explanation is useless, unscientific and must be discarded?

What happens to empirical work? Scheel et al. refer to research into kama muta as a distinct "heart-warming emotion" and laud the investigators' attention to detail, multi-method exploratory work, the effort that is put into creating a validated multi-lingual instrument and careful selection of reliable stimuli. A key question the investigators wish to answer is whether the emotion can be consistently and uniquely invoked when people are reminded of their connection to others; they theorize that kama muta may have an adaptive function as it encourages communal sharing relationships. In our opinion this is serious and valuable research, but if Scheel et al. insist that we consider it as the explorative precursor to a hypothetico-deductive endeavor then all hope is lost: a yes/no hypothesis loosely tied to a vague adaptive explanation is about as far away from a bold theory-driven conjecture as scientifically possible, with no obvious recourse.

Questionable research practices can be dealt with, predictions can be sharpened, statistics and psychometrics treated with renewed deference. The bar can be set higher, this much is uncontroversial. But what if competing theories lead to similar causal models? What if we simply cannot predict the magnitude of an effect with even the most sophisticated of theories? What if it's unclear when a phenomenon will or won't show up? If we embrace the harsh requirements of hypothetico-deductive science and demand conclusive experiments, the honest answer is that we must refuse to investigate questions that cannot currently be translated into hypotheses that are unescapable consequences of a well-defined theory and that cannot produce bold predictions that unambiguously differentiate between competing theories. "Do we know how anxiety changes intention, how memories alter decisions, how intelligence changes emotion, and so on? And, of course, has anyone ever explained how the mind works on the body or the body on the mind? Questions of that sort should never have been asked." (Skinner $1987,786)$

In choosing the path of hypothetico-deductive research, we would also have to accept that whatever questions do permit of a rigorous theory-based answer may prove unable to assist in answering the fuzzier psychological questions a more applied companion discipline might ask. Universities house plenty of disciplines that study more or less the same thing at different 
levels of abstraction yet seem unable to help each other. In the natural sciences, the behavior of subatomic particles does not determine the chemical properties of atoms and molecules (Hossenfelder 2018, ch. 3). Chemistry has next to no bearing on cell biology - a key insight that sets modern molecular biology apart from earlier biochemistry (Judson 2013, 321-22). Cell biology in turn is of limited importance to agriculture. Classical optics, which ignores the particle-wave duality of photons, is alive and well. Hydraulics, the engineers' study of liquids, coexists with and is quite different from the physical subfield of hydrodynamics. The little amount of foundational theory we do have in psychology seems to show a similar separation of scales (as physicists would call it): Thorndike's operant conditioning is only vaguely useful to clinical psychology, Ebbinghaus' forgetting curve only marginally relevant to educational science. Perhaps hypothetico-deductive psychology will be different, but thus far attempts to refashion psychology into a purer, more theoretical enterprise have tended to be programmatic, with few motivating examples to show how such a transformation would retain or would inform current questions.

For those among us who care about the variety of questions that fascinate psychologists today and do not wish to wait around for a tough-minded theoretical foundation that may never arrive (or could prove esoteric if it does), it is worth asking how we might go about answering psychological questions using plebeian induction instead: how would it affect our methods, our experiments, the way we interpret evidence?

\section{More data, from more populations, in more settings}

In order to learn about the world, we may explore and document the great variety of phenomena, to organize them, to see whether there are any obvious regularities or striking differences between different people, geographies, times. What makes hypothetico-deductive science so special is that it allows us to dispense with such natural history: when we make bold predictions and test whether they pan out, we will learn much about the world without having to become a collector or a taxonomist. Alas, if the hypothesis-driven approach proves impossible for the kinds of questions that arouse our interest, we must revert to this older kind of science, and laboriously collect and analyze what is out there.

In meteorology, a disdrometer measures the size of rain drops. Measurements have been taken all across the world, across the seasons, during light drizzles, normal rainfall and during storms, when it rains but also when it snows or hails. On the basis of this data, various curve fitting exercises have been performed, the most well-known of which is the Marshall-Palmer equation. The equation is known as an "empirical law," that is, it has nothing to do with the viscosity of water, acceleration due to gravity or any other fundamental physical desideratum, but it happens to provide a good-enough approximation for the distribution of rain drop size given a particular intensity of precipitation. It has proven a useful building block to calculate the amount of wind-driven rain to expect on a building facade (and thus how diligently it must be weatherproofed) and to interpret data from weather radars, which we use for so-called nowcasts of current and upcoming weather.

Zoology, botany and mycology are not known to be the most dynamic endeavors and at times have found it hard to attract talented scientists (Mayr 1963), but the description, categorization, anatomy and physiology of over a million species of plants, animals, fungi, bacteria and other simple organisms surely must count as one of the crowning scientific achievements of our times. 
In materials science, thousands of substances are measured for their compressive strength, tensile strength, hardness, elasticity, thermal conductivity, thermal expansion, heat capacity, electromagnetic conductivity, viscosity, reflectivity and much more, and materials are developed to meet new requirements for any of these properties. Physicists have helped to develop many of the workhorses of the field, from scanning tunneling microscopes to diamond anvil cells, but physics has been no help in predicting the behavior of these materials. Silver bromide photography was not understood chemically or physically until roughly a century after it was invented (Gurney and Mott 1938). The chemical properties of graphene, for which Andre Geim and Konstantin Novoselov received a Nobel prize in 2010, were a surprise to everyone, and this despite the maturity of crystallography.

There is an incredibly utility in just knowing the facts and organizing them. Henri Poincaré is often quoted as saying that science is not just a jumble of facts and this is taken as a defense of theoretical science. But what he writes immediately before that famous quote is quite different: le savant doit ordonner. (Poincaré 1902, 168) This is something that is alien to contemporary experimental psychology, but is not alien to the discipline, historically speaking (Cronbach 1957).

Consider the studies into productivity conducted between 1927 and 1932 at a Western Electric factory outside of Chicago, the Hawthorne Works, by Elton Mayo and his graduate assistant Fritz Roethlisberger. They had planned a number of straightforward interventions that would vary (for example) the amount of light in some but not other factory workshops and they would record any changes in productivity, such as the number of electromagnetic coils a worker might wind per hour. But the investigators quickly found out that "the results obtained, instead of giving definite answers to the original questions, demanded a restatement of them." They changed tack and considered the need to know much more about factory life before jumping to conclusions, and realized that they would have to let the facts speak for themselves, using theories and hypotheses only as "ways of looking at facts which assisted the research investigators to find more facts or to make more adequate discriminations in fact." (Roethlisberger and Dickson 2004, 5:2-3) The depth and length of these investigations, recorded in a tome that runs to over six hundred pages, the willingness to record not just whatever variables one would need for a tabulation but any salient observations encountered along the way, and the insistence on both artificial manipulations and the need to study real people doing real work, have been an inspiration for industrial and organizational psychologists ever since.

Lewis Terman's Genetic Studies of Genius, in which gifted children were followed up well into adulthood, consists of data and interviews collected over more than fifty years. Terman wished to know whether common stereotypes of the gifted held any truth: were they more sickly, less social, bound to become unhappy but brilliant scientists and statesmen - empirical questions, valuable in their own right, and hypotheses as to whether and why these traits go together are secondary. Terman also anticipated that there might be no straightforward answers, and that interviews and correspondence with participants would be needed to give context to the numbers - an empirical rather than a theoretical attitude to research.

Finally, we may marvel at the impact achieved by Alfred Kinsey's surveys of sexual behavior in the fifties. When psychologists and psychiatrists realized that homosexuality was far more common than previously thought, that made it harder to pathologize and categorize as aberrant 
behavior, ultimately leading to its expulsion from the Diagnostic and Statistical Manual of Mental Disorders (Chiang 2008). No hypothesis, no theory required.

As with most older studies, these examples have their flaws. Some of the Hawthorne experiments failed to maintain a proper control arm and are hopelessly confounded (Levitt and List 2009); Terman's conclusions were biased by his belief in eugenics; and it's not clear why Kinsey felt that it was important for science to invite co-workers to his attic for sex but not important to weight his survey samples. Yet these studies also remind us how groundbreaking it can be to just know the facts (Eronen and Bringmann 2021)!

\section{More experiments and more than experiments}

In physics, cunning experimental setup is often necessary to bring elusive phenomena to the fore. Theories as different as night and day may nevertheless make identical predictions under a wide range of conditions and differ only on the fringes, which means not just any experiment will be able to differentiate winner from loser.

Benjamin Franklin's attempts to figure out the secret workings of electricity at one point involved electrocution of turkeys, while like-minded scientists toyed around with Leyden jars, induction coils and all manner of odd contraptions.

It took physicists over 40 years to conceptualize, engineer and build the experimental apparatus required to verify the existence of Peter Higgs' conjectured elemental particle, the Higgs boson. A particle accelerator now tunnels under France and Switzerland, measures 26.659 meters in circumference, consumes as much electricity as a small city and smashes particles into each other at very near the speed of light.

Educational psychologists, similarly, might test whether adding a bit more struggle to instructional materials and slowing down students aids their understanding not through something a teacher might actually try to implement, for example changes to a curriculum that encourage slow, deliberate processing, but instead by comparing the performance of students who receive regular course materials with the performance of students who receive materials identical in content but printed in a grayed out and hard to read font. Whether or not a teacher or publisher would ever consider intentionally obfuscating course materials in such a fanciful manner is besides the point. Ecological veracity of the intervention is an irrelevant concern for the theoretically minded researcher, just as it would be for the physicist and her particle accelerator or the geneticist and his fruit flies: it's a good intervention if it's a good test of our theory.

When testing a theory, there is no requirement for test subjects and manipulation to be anything that is intrinsically interesting. Instead, experiments serve to confirm a concept, hypothesis or theory and we should use manipulations that foreground the defining features of our theory, with an outcome that cannot easily be guessed and that hopefully provides a unique contrast with competing theories. Measurements hold no value outside of that confirmatory context.

Unusual and cunning interventions, then, are par for the course in deductive science. They are in fact often the only way in which we can properly grill a theory. The natural sciences' academic literature is full of poetic phrases to this end: we must interrogate nature, we must 
trick nature into giving up its secrets, we must design experiments that let us peek behind nature's curtains!

However, studying one thing in order to learn about another is a dangerous bet, and a bet that does not make sense outside of hypothetico-deductive science. If information from this specific intervention does not readily flow upstream to the theory at test (and we have argued that it does not), and from there once more downstream to the entire class of problems that this theory might inform (which depends very much on how strongly a theoretical discipline and its more applied cousin are interfaced, some are but many are not), the situation is not very different from testing whether painting purple dots on rhinoceros in Mozambique affects soil quality in Nunavut. In the absence of greater motive, fanciful interventions are no longer science, they are nonsense.

Thus, a confusingly worded hypothetical scenario about a bank teller called Linda is supposed to provide deep insight into human reasoning (Tversky and Kahneman 1982), surprisingly high test results for students who learned from bad photocopies point the way to an educational revolution (Diemand-Yauman, Oppenheimer, and Vaughan 2011) and if kids who play violent video games are afterwards more likely to subject their competitors to loud noises than nongamers, that's a perfectly unobjectionable proxy for physical violence and you'd better watch out (Engelhardt et al. 2011)!

Such psychological research ends up being useless because - with vague predictions on the basis of a hypothesis that is only loosely connected to a theory that can be interpreted any which way - it can not provide strong confirmation of a theoretical construct, but neither does it provide empirical material that carries much weight in an inductive argument. The epistemic requirements of inductive science and deductive science are polar opposites: one demands a lot of data, captured from a lot of angles, involving a lot of people, in a lot of situations, in a lot of places; the other is impressive precisely because all it needs is a tiny sliver of empirical input from a well-chosen but idiosyncratic angle.

Psychological experiments whose prime concern is to corroborate or falsify a theory run the risk of becoming weird or zany, an inside game where researchers try to outdo each other with ever more outlandish experimental setups (Ring 1967). Such artifice is not intrinsically foolish, but in psychology it is rarely insightful.

Anthropologist Roy D'Andrade astutely observed that while experimental psychology is a hotbed of scientific activity, it is unclear "the degree to which the findings of the laboratory have any relation to the things that people do in ordinary life, the degree to which findings represent historically conditioned cultural attitudes rather than deep psychological process, and the way in which the exploration of a particular phenomenon seems to lead to a dead end, with no general findings outside of the understanding of how to produce a certain limited class of effects." (D'Andrade 1986, 37)

Psychologists have not been blind to the issue. Hence the rich literature and recurring worries among researchers about ecological validity. Consider, however, that such worries do not make any sense in a theoretical science, where the only demand made of a cunning experiment is that it is revealing, not that it is realistic. Insofar as psychologists do worry about the ecological validity of lab experiments, they implicitly acknowledge the shortcomings of the hypothesis-driven approach. 
Experiments in the applied sciences and other inductive disciplines are much more wary of ceteris paribus assumptions.

When structural engineers wish to test the crack resistance of different formulations of concrete and different steel reinforcements, they will test exactly that and preferably in conditions that resemble how the concrete will be employed in real building projects. They will take into account chemical knowledge about how different additives interact, and will use that knowledge to test the most promising candidates first, but they won't take for granted that some formulations absolutely can or cannot work.

When food scientists want to know how well people tolerate chocolate that substitutes a lowcalorie sweetener such as steviol glycoside for sugar, they will prepare a batch of reformulated chocolate and perform a blind triangle test against the original, and it would never occur to these scientists that they could rely on the results from a colleague's report on flavor perception of aspartame in vanilla cupcakes, let alone anatomical results about what taste receptors steviol glycoside will and won't bind to.

Along those lines, when psychologists wish to learn more about altruism and fairness they have found it useful to supplement observational studies of moral behavior with stylized experiments such as the ubiquitous ultimatum game, where one participant decides how much of a sum of money to share with another participant while the other decides if that amount of money is fair and if not, neither receives anything. In a variant, the dictator game, the second participant has no agency and must accept the offer. An empirically minded psychologist would value such experiments, too: they isolate and highlight aspects of human behavior not clearly visible in the field. But for an empiricist such experiments are merely one source of evidence among many and not in any way superior to ethnology, historical or sociological research. An empirically minded psychologist would also insist we repeat the experiment with subjects of different ages, social and cultural backgrounds. Some behaviors are universal, but not all. Under the auspices of Joseph Henrich many such repeats have taken place, and have shown great heterogeneity in perceptions of fairness across cultures (Henrich, Heine, and Norenzayan 2010). Unsurprisingly, Henrich is an anthropologist, a discipline known for its meticulous field research and an appreciation of what makes each culture different.

\section{Final thoughts}

Just about every freshman student of psychology is wowed when they first hear about the invisible gorilla. Such a simple, elegant setup, and it seems to offer an important glimpse into the nature of human attention.

Likewise, isn't it marvelous that if we ask research subjects to solve a language task involving the words rude, bother, brazen and obnoxious, we find that later they're apt to interrupt people?

Unfortunately, cunning experiments designed with the sole purpose of corroborating a theory do not age well. For some of these experiments, attempts at replication show that their findings do not apply beyond the college student demographic, do not generalize to stimuli that ought to be equivalent, or turn out to be a statistical fluke. For others, it's not clear why these 
unusual interventions matter at all. Why would we expect people to see the gorilla in Chabris and Simmons' famous experiment, given that participants have been explicitly instructed to concentrate on counting basketball passes and nothing else? What did we prove other than the existence of attention? (Gigerenzer 2018)

Empirical science is not just a different kind of science, it's also a humbler attitude towards science.

Psychologists have long assumed that the point of an experiment is to prove a theory right or wrong. As empirical scientists, instead we start our investigations from the assumption that "if a person of reasonable competence and seriousness states a hypothesis about a human social phenomenon, then it is almost inevitably valid" (McGuire 2013, 417) - not everywhere, for everyone, but somewhere and for some people. Such hypotheses are too weak to support much theory, but they can still help us find mechanisms, "frequently occuring and easily recognizable causal patterns that are triggered under generally unknown conditions or with indeterminate consequences." (Elster 2015, 26) This leads to a very different kind of research wherein we don't prove or disprove theories, but rather try to find the conditions under which a particular phenomenon or mechanism will or won't show up, what strengthens and weakens it (Greenwald et al. 1986). Through slow, careful mapping of the territory, we will start to see whether a behavioral or cognitive phenomenon is widespread, robust or ephemeral, whether it strongly affects our actions or life outcomes, or whether is only a curiosity with limited impact.

Ours is not the only proposal floating around to make psychology a more reliable science. We probably do, as a discipline, need to be a bit more strict $(\mathrm{p}<.005)$, a bit more honest (preregistration) and a bit more diligent (replication, meta-analysis) (Daniel et al. 2018; Nosek et al. 2018; Ioannidis 2014). However, all such proposals still consider the cunning, confirmatory and conclusive experiment to be the engine for scientific progress in psychology. If the hypothetico-deductive engine itself is broken, what first appear to be hard-hitting proposals, to the point of self-flagellation, may offer no more than marginal improvements over the status quo. Greater strictness does not catch unwarranted generalizations, and neither do replications (Yarkoni 2020). Preregistered experiments do not produce decisive evidence in favor of one theory or another, because that's simply not something psychological hypotheses are capable of. Curbing questionable research practices does nothing when accepted research practices are ineffectual. First and foremost we must recalibrate our ideas of what the science of mind and behavior ought to be like, only then does it make sense to police scientific norms. Saying farewell to the cunning, confirmatory and conclusive experiment of hypothetico-deductive science may well provide the missing piece of the puzzle.

\section{Acknowledgements}

We would like to thank Julia Rohrer and Tal Yarkoni for feedback on earlier versions of this manuscript. We would also like to thank Joe Cesario, Wolf Vanpaemel and an anonymous reviewer for their instructive comments. 


\section{Bibliography}

Blum, Ben. 2018. "The Lifespan of a Lie." Gen. https://gen.medium.com/the-lifespan-of-alie-d869212b1f62.

Borghi, Anna M., and Chiara Fini. 2019. "Theories and explanations in psychology." Frontiers in Psychology 10 (APR): 1-3. https://doi.org/10.3389/fpsyg.2019.00958.

Bullock, John G., Donald P. Green, and Shang E. Ha. 2010. "Yes, But What's the Mechanism? (Don't Expect an Easy Answer)." Journal of Personality and Social Psychology 98 (4): 550-58. https://doi.org/10.1037/a0018933.

Burghardt, Juliane, and Alexander Neil Bodansky. 2021. "Why Psychology Needs to Stop Striving for Novelty and How to Move Towards Theory-Driven Research." Frontiers in Psychology 12 (January). https://doi.org/10.3389/fpsyg.2021.609802.

Cahalan, Susannah. 2019. The Great Pretender: The Undercover Mission That Changed Our Understanding of Madness. Grand Central Publishing.

Campbell, Donald T. 1979. "A Tribal Model of the Social System Vehicle Carrying Scientific Knowledge." Science Communication 1 (2): 181-201. https://doi.org/10.1177/1075547079 00100202.

Chiang, Howard Hsueh-hao. 2008. "Effecting Science, Affecting Medicine: Homosexuality, The Kinsey Reports, And The Contested Boundaries Of Psychopathology In The United States, 1948-1965." Journal of the History of the Behavioral Sciences 44 (4): 300-318. https://doi.org/10.1002/jhbs.

Coles, Peter. 2019. "Einstein, Eddington and the 1919 eclipse." Nature 568 (7752): 306-7. https://doi.org/10.1038/d41586-019-01172-z.

Cronbach, Lee J. 1957. "The Two Disciplines of Scientific Psychology." American Psychologist 12 (11): 671-84. https://doi.org/10.1007/s10440-009-9506-5.

D'Andrade, Roy. 1986. "Three Scientific World Views and the Covering Law Model." In Metatheory in Social Science: Pluralisms and Subjectivities, 19-41. Chicago: University of Chicago Press.

Daniel, J, O James, A Brian, A Kenneth, D Christopher, D Thomas, De Boeck, et al. 2018. "Redefine Statistical Significance." Nature Human Behaviour 2, 6-10. https://doi.org/10.1 038/s41562-017-0189-z.

Diemand-Yauman, C., D. M. Oppenheimer, and E. B. Vaughan. 2011. "Fortune favors the bold (and the Italicized): effects of disfluency on educational outcomes." Cognition 118 (1): 111-15. https://doi.org/10.1016/j.cognition.2010.09.012.

Elster, Jon. 2015. Explaining Social Behavior: More Nuts and Bolts for the Social Sciences. Cambridge University Press.

Engelhardt, Christopher R., Bruce D. Bartholow, Geoffrey T. Kerr, and Brad J. Bushman. 2011. "This is your brain on violent video games: Neural desensitization to violence predicts 
increased aggression following violent video game exposure." Journal of Experimental Social Psychology 47 (5): 1033-36. https://doi.org/10.1016/j.jesp.2011.03.027.

Eronen, Markus I., and Laura F. Bringmann. 2021. "The Theory Crisis in Psychology: How to Move Forward." Perspectives on Psychological Science. https://doi.org/10.1177/174569 1620970586.

Fried, Eiko I. 2020. "Lack of Theory Building and Testing Impedes Progress in The Factor and Network Literature." Psychological Inquiry 31 (4): 271-88. https://doi.org/10.1080/10 47840X.2020.1853461.

Gigerenzer, Gerd. 2010. "Personal Reflections on Theory and Psychology." Theory \& Psychology 20 (6): 733-43. https://doi.org/10.1177/0959354310378184.

—. 2018. "The Bias Bias in Behavioral Economics Gerd Gigerenzer," 303-36. https: //doi.org/10.1561/105.00000092.

Greenwald, Anthony G., Anthony R. Pratkanis, Michael R. Leippe, and Michael H. Baumgardner. 1986. "Under What Conditions Does Theory Obstruct Research Progress?" Psychological Review 93 (2): 216-29.

Griggs, Richard A., and Andrew N. Christopher. 2016. "Who's Who in Introductory Psychology Textbooks: A Citation Analysis Redux." Teaching of Psychology 43 (2): 108-19. https://doi.org/10.1177/0098628316636276.

Gurney, R. W., and N. F. Mott. 1938. "The Theory of the Photolysis of Silver Bromide and the Photographic Latent Image." Proceedings of the Royal Society of London. Series A, Mathematical and Physical Sciences 164 (917): 151-67.

Henrich, Joseph, Steven J Heine, and Ara Norenzayan. 2010. "The weirdest people in the world?" The Behavioral and Brain Sciences 33: 61-83; discussion 83-135. https: //doi.org/10.1017/S0140525X0999152X.

Hossenfelder, Sabine. 2018. Lost in Math. New York: Basic Books.

Ioannidis, John P. a. 2014. "How to Make More Published Research True." PLoS Medicine 11 (10): e1001747. https://doi.org/10.1371/journal.pmed.1001747.

Judson, Horace Freedland. 2013. The Eighth Day of Creation. Cold Spring Harbor, New York: Cold Spring Harbor Laboratory Press.

Laudan, Larry. 1981. "Hume (and Hacking) on Induction." In Science and Hypothesis, 72-85. Springer Netherlands. https://doi.org/10.1007/978-94-015-7288-0_6.

Levitt, Steven D., and John A. List. 2009. "Was There Really a Hawthorne Effect At the Hawthorne Plant? an Analysis of the Original Illumination Experiments." https: //arxiv.org/abs/arXiv:1011.1669v3.

Lykken, David T. 1991. "What's wrong with psychology anway?" In Thinking Clearly about Psychology, edited by Dante Cicchetti and William M. Grove, 1:1-13. Minneapolis, Minnesota, USA: University of Minnesota Press. https://doi.org/10.1016/0272-7358(92)9 0024-3. 
Mayr, Ernst. 1963. "The New versus the Classical in Science." Science 141 (3583): 765.

McGuire, William J. 2013. "An Additional Future for Psychological Science." Perspectives on Psychological Science 8 (4): 414-23. https://doi.org/10.1177/1745691613491270.

McPhetres, Jonathon, Nihan Albayrak-Aydemir, Ana Barbosa Mendes, Elvina C. Chow, Patricio Gonzalez-Marquez, Erin Loukras, Annika Maus, et al. 2021. "A decade of theory as reflected in Psychological Science (2009-2019)." PLoS ONE 16 (3 March 2021): 12-14. https://doi.org/10.1371/journal.pone.0247986.

Meehl, Paul E. 1978. "Theoretical risks and tabular asterisks: Sir Karl, Sir Ronald, and the slow progress of soft psychology." Journal of Consulting and Clinical Psychology 46 (113): 806-34.

Mischel, W. 2008. "The toothbrush problem." APS Observer 21 (11).

Muthukrishna, Michael, and Joseph Henrich. 2019. A problem in theory. Vol. 3. 3. https://doi.org/10.1038/s41562-018-0522-1.

Nosek, Brian A., Charles R. Ebersole, Alexander C. DeHaven, and David T. Mellor. 2018. "The preregistration revolution." Proceedings of the National Academy of Sciences of the United States of America 115 (11): 2600-2606. https://doi.org/10.1073/pnas.1708274114.

Oberauer, Klaus, and Stephan Lewandowsky. 2019. "Addressing the theory crisis in psychology." Psychonomic Bulletin and Review 26 (5): 1596-1618. https://doi.org/10.3758/s13423019-01645-2.

Open Science Collaboration. 2015. "Estimating the reproducibility of psychological science." Science 349 (6251). https://doi.org/10.1126/science.aac4716.

Perry, Michael F. 2007. "Remembering the oil-drop experiment." Physics Today 60 (5): 56-60. https://doi.org/10.1063/1.2743125.

Platt, John R. 1964. "Strong Inference." Science 146 (3642): 347-53.

Poincaré, Henri. 1902. La Science et l'Hypothèse. Paris.

Rautio, James. 2014. "The Long Road to Maxwell's Equations." https://spectrum.ieee.org/te ch-history/dawn-of-electronics/the-long-road-to-maxwells-equations.

Ring, Kenneth. 1967. "Experimental Social Psychology: Some Sober Questions about Some Frivolous Values." Journal of Experimental Social Psychology 3: 113-23.

Roberts, Seth, and Harold Pashler. 2000. "How persuasive is a good fit? A comment on theory testing." Psychological Review 107 (2): 358-67. https://doi.org/10.1037//0033295X.107.2.358.

Roethlisberger, F. J., and W. J. Dickson. 2004. Management and the Worker. Vol. 5. Routledge.

Scheel, Anne M., Leonid Tiokhin, Peder M. Isager, and Daniël Lakens. 2020. "Why Hypothesis Testers Should Spend Less Time Testing Hypotheses." Perspectives on Psychological Science. 
Schimmack, Ulrich. 2012. "The ironic effect of significant results on the credibility of multiplestudy articles." Psychological Methods 17 (4): 551-66. https://doi.org/10.1037/a0029487.

Skinner, B. F. 1987. " "Whatever happened to psychology as a science of behavior?"." American Psychologist 42 (8): 780-86. https://doi.org/10.1037/0003-066x.43.10.824.

Stoney, G. Johnstone. 1894. "Of the 'electron,' or atom of electricity." Philosophical Magazine 38 (233): 418-20. https://doi.org/10.1080/14786449408620653.

Tversky, Amos, and Daniel Kahneman. 1982. "Judgments of and by representativeness." In Judgment Under Uncertainty, edited by D. Kahneman, P. Slovic, and A. Tversky, 84-98. Cambridge University Press. https://doi.org/10.1017/CBO9780511809477.007.

Wade, Nicholas. 1982. "Smart Apes, or Dumb?"

Wigner, Eugene P. 1960. "The unreasonable effectiveness of mathematics in the natural sciences." Communications On Pure And Applied Mathematics 13: 1-14. https://doi.org/ 10.1179/030801811X13082311482537.

Yarkoni, Tal. 2020. "The Generalizability Crisis." Behavioral and Brain Sciences, 1-37. https://doi.org/10.31234/osf.io/jqw35. 